\title{
Room Temperature Serial Crystallography for the Masses: How Structural Biologists Can Benefit from Crystallographers' Newest Toys
}

\author{
Aaron D. Finke ${ }^{a}$ \\ a MacCHESS, Cornell University, Ithaca, NY, USA af554@cornell.edu
}

Protein crystallography enables atomic-level structure determination from crystalline specimens. Due to the rapid onset of radiation damage at room temperature, the vast majority of protein crystal structures are done at cryogenic temperatures. While cryocooling alleviates much of the practical issues of radiation damage, it also restricts the energy landscapes in which proteins normally exist, which can lead to inaccurate pictures on the room temperature dynamics of conformation, allostery, and substrate binding. ${ }^{[1]}$

Crystallography at room temperature is restricted by the rapid onset of radiation damage. One of the most effective means of overcoming this obstacle is to collect data "serially": that is, collect a small amount of data from each crystal and merge it together. We have developed a serial crystallography program at CHESS for users interested in collecting room temperature data. Silicon chips with microwell features enable high throughput, while the use of oscillation reduces the total number of crystals needed for a full dataset. An optimized data processing protocol maximizes dataset quality while minimizing the number of crystals needed.
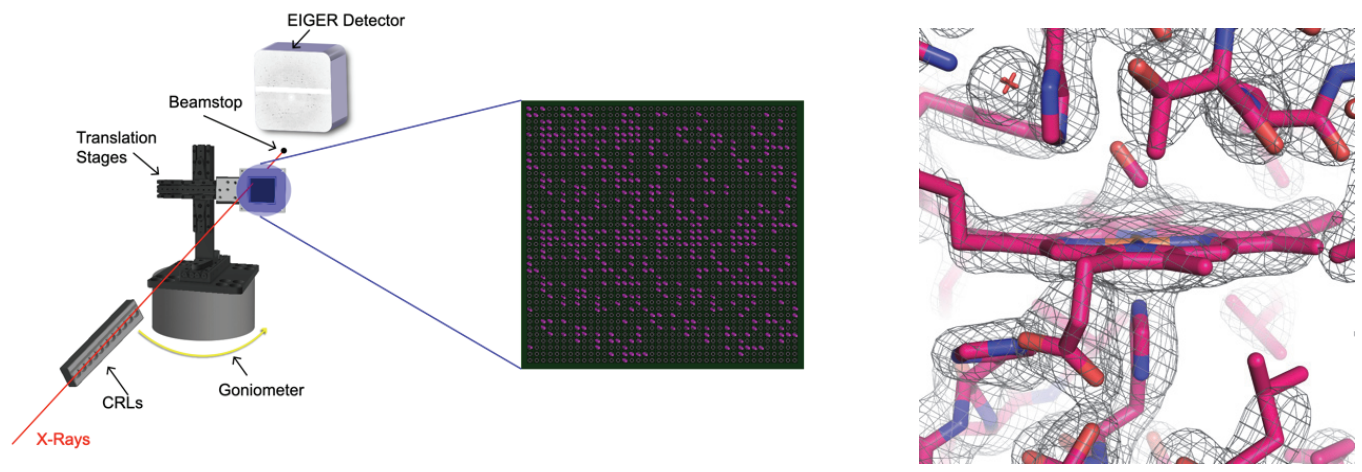

References

[1] see e.g. Keedy, D. A. (2019) Acta Cryst., D75, 123-137.

[2] Wierman, J. L.; Paré-Labrosse, O.; Sarracini, A.; Besaw, J. E.; Cook, M. J.; Oghbaey, S.; Daoud, H.; Mehrabi, P.; Kriksunov, I.; Kuo, A.; Schuller, D. J.; Smith, S.; Ernst, O. P.; Szebenyi, D. M. E.; Gruner, S. M.; Miller, R. J. D.; Finke, A. D. "Fixed-Target Serial Oscillation Crystallography at Room Temperature," IUCrJ, 6, 305-316. 